\title{
SIZE-DEPENDENT THERMODYNAMIC AND ELECTRONIC PROP- ERTIES OF INDIVIDUAL NANOMETER-SIZE SUPPORTED GOLD CLUSTERS
}

\author{
M.E. LIN," A. RAMACHANDRA,"* R.P. ANDRES"* and \\ R. KEIFENBERGER* \\ Purdue University \\ - Department of Physics FGO2-84ER 45162 \\ ** School of Chemical Engineering \\ W. Lafayette, IN 47907 \\ U.S.A.
}

ABSTRACT. Fieit emission techniques devised to measure the melting temperature and electronic struc'ure of individual, nanometer-size clusters supported on electrically conducting substrates are reviewed. Data on the size-dependent reduction in melting temperature of Au clusters are compared to existing thermodynamic descriptions and molecular dynamic calculations. Data on the electronic structure of an individual $1 \mathrm{~nm}$ Au cluster are compared to the predictions of simple electron shell models for cluster electronic staies.

\section{Introduction}

A recent focus in cluster research is the novel structural, electronic, and optical properties expected for cluster-assembled, nanophase materials. ${ }^{1-3}$ To date most information on the properties of small clusters has been obtained from cluster-beam experiments in which isolated clusters are studied while in flight. This research has established that in the nanometer size range, unsupported or free-space clusters exhibit unique, size-dependent, structural and electronic features different from bulk samples of the same atoms, but cannot answer important questions regarding their properties for cluster-assembled materials. An important research approach that bridges the gap between studies of free clusters and cluster-assembled materials is the study of an individual cluster supported on a solid surface. The information obtained from such single-cluster studies is of fundamental importance to an understanding of the interaction between clusters and substrates and for determining whether the unique properties of free clusters survive when they are deposited on a solid surface. By performing experiments on a single supported cluster, uncertainties associated with

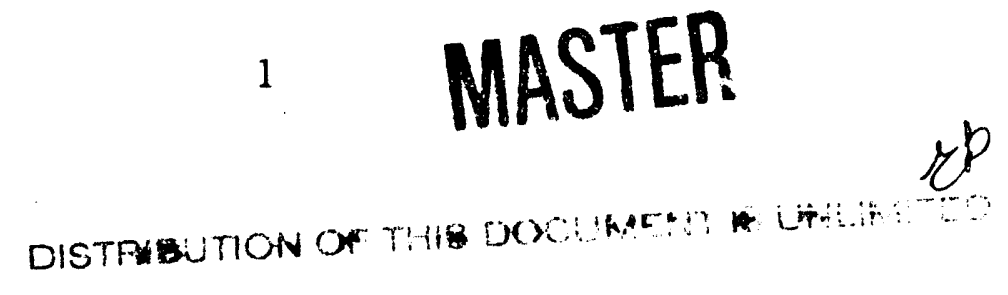


the size distribution of clusters, that plague experiments requiring an ensemble of supported clusters, can be circumvented.

The following paper reviews experimental techniques recently developed for studying individual supported clusters and summarizes results that have been obtained in our laboratory using these techniques. ${ }^{4.5}$ Controlled-size clusters grown in a multiple expansion cluster source (MECS), ${ }^{6}$ and subsequently deposited on clean substrates, have been used to study melting temperature as a function of cluster size, ${ }^{7-9}$ the shape of supported Au clusters on flat substrates, ${ }^{10,11}$ and the size-dependent electronic structure of supported Au clusters. ${ }^{12}$

\section{Field Emission Techniques}

The field emission process is a well known phenomenon that relies on the pointprojection imaging of electrons emitted from a small apex of an electrically conducting tip subjected to a high electric field. The field emission process has been well studied from a theoretical context and has been used to provide a wide variety of information about the work function of metal surfaces, ${ }^{13}$ surface diffusion of adsorbates on metallic surfaces, ${ }^{14,15}$ the electronic band structure of metal surfaces near the Fermi energy, ${ }^{16}$ and the interaction of light with sub-micron metallic surfaces. ${ }^{17,18}$

Field emission experiments are versatile and relatively simple to conduct. The common ingredients in any field emission experiment include a sharp metallic tip positioned in front of a light emitting screen located inside an ultra-high vacuum (UHV) chamber. The tip can be constructed from a large variety of high meltingpoint elements, with tungsten being the material of choice for most studies. The tips are usually electro-chemically etched from a wire in much the same way that scanning tunneling microscope tips are often prepared. ${ }^{18}$ This etching produces a rough, irregular tip with an end form of radius $\sim 10-50 \mathrm{~nm}$. An important modification occurs to the tip morphology when it is mounted in a UHV chamber and heated repeatedly to high temperatures to produce an atomically clean surface. The heating procedure produces a well-annealed end form of radius $\sim 100 \mathrm{~nm}$ that is single crystal faceted along crystallographic directions with low surface free energy. Thus, the tip that supports the clusters in our studies is a well annealed single crystal whose orientation can be readily discerned from the syrnmetry of the resulting field emission pattern.

The key that makes field emission techniques useful to study supported clusters is the small radius of curvature of the supported cluster when compared to the underlying field emission tip. This simple geometric consideration results in $n$ electric field enhancement around the cluster with respect to the substrate and in win results in electron emission from the cluster alone.

To produce a measurable field emission current usually requires a few thousand volts potential difference between the tip and a nearby phosphor-coated screen $(\sim 10$ 
$\mathrm{cm}$ diameter) located in front of the field emission tip. The image of the field emitted electrons can then be viewed directly from the screen. Often, it is convenient to store the field emission images on a video recorder for further analysis. More quantitative measurements can be made by measuring the current as a function of the applied voltage or by energy resolving the emitied electrons with a suitable field emission energy analyzer.

The field emission process has been well studied and is concerned with the calculation of $j(E)$, the number of electrons of mass, $m$, emitted per unit area per unit time with energy between $E$ and $E+d E$, due to an electric field $F$ applied in the 2-direction. ${ }^{16}$ This calculation requires an integration over all possible energies $E$ and wavevectors $\vec{k}=\left(k_{z}, k_{\|}\right)$

$$
j(E) d E=\int_{E}^{E+d E} N(\vec{k})\left|T\left(k_{2}, E\right)\right|^{2} \frac{d^{3} k}{(2 \pi)^{3}}
$$

where, $N(\vec{k})$ is the number of electrons with wavevector $\vec{k}$ incident on the barrier per unit time per unit area and $\left|\mathrm{T}\left(\mathrm{k}_{\mathrm{z}}, \mathrm{E}\right)\right|^{2}$ is the transmission probability through the surface potential barrier. The metal-vacuum interface is often described in 1 dimension by an image rounded barrier given by

$$
\begin{aligned}
V(z) & =E_{F}+\phi-\frac{e^{2}}{4 z}-e F z & & \text { for } z>z_{c} \\
& =0 & & \text { for } z<z_{c}
\end{aligned}
$$

where $z_{c}$ is determined by $V\left(z_{c}\right)=0, E_{F}$ is the Fermi-energy of the metal and $\phi$ is the metal work function (see Figure 1).

Using the WKB approximation to estimate $T\left(k_{z}, E\right)$ and a free electron approximation to evaluate $N(\vec{k})$, the distribution of emitted electrons is:

$$
j_{0}(E)=\frac{m d_{E} f(E)}{2 \pi^{2} \hbar^{3}} e^{-\frac{4}{3}\left(\frac{2 m}{\hbar^{2}}\right)^{\frac{1}{2}} \frac{\left(\phi+E_{F}-E\right)^{\frac{3}{2}}}{e F} v(y)}
$$

where

$$
\frac{1}{d_{E}}=2\left(\frac{2 m}{\hbar^{2}}\right)^{\frac{1}{2}} \frac{\left(\phi+E_{F}-E\right)^{\frac{1}{2}}}{e F} t(y)
$$

$v(y), t(y)$ are slowly varying, tabulated ${ }^{20}$ functions of $y=\left(e^{3} F\right)^{\frac{1}{2}} /(\phi)$ that account for the static image-rounded term in the potential given in Eq. 2, and $f(E)$ is the Fermi-Dirac function describing the distribution of filled states within the metal.

The shape of $j_{0}(E)$ is roughly governed by the temperature of the emitter for $E>E_{F}$ and the rapid decrease in the transmission probability through the metalvacuum interface for $E<E_{F}$. The exponentially rapid decrease in the transmission probability with energy below $E_{F}$ strongly favors electron emission from filled states nearest the Fermi energy. An experimental measurement of this energy distribution 


\section{Metal-Vacuum Potential Barrier}

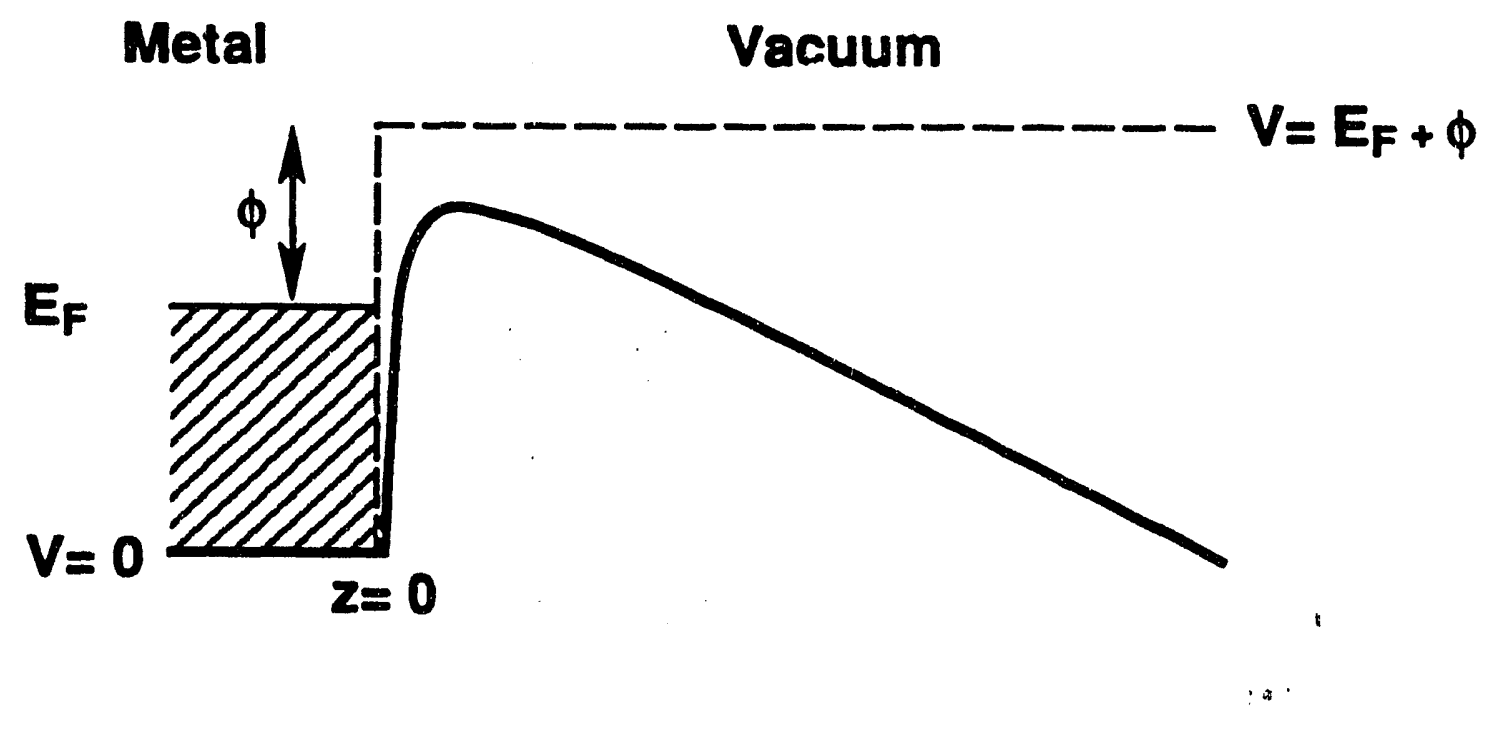

Figure 1: A simple model for the surface potential barrier of a metal-vacuum interface described by Eq. 2. The barrier of finite width is a result of an applied electric field. Electrons tunneling from the metal through the barrier into the vacuum produce a field emission energy distribution given by $\mathrm{Eq}$. 3. When a nanometer-size metallic cluster is deposited on the metal substrate, electrons tunneling from the quantized cluster states can be measured. 
involves further convolution of Eq. 3 with a symmetric gaussian window function that accounts for the finite energy resolution of the energy analyzer. It is an experimental fact that Eq. 3 well describes the overall shape of the distribution of electrons emitted from field emission tips fabricated from a variety of refractory metals. ${ }^{16}$

Eq. 3 can be further integrated to give the total current

$$
\begin{aligned}
J(F) & =e \int_{-\infty}^{\infty} j_{0}(E) d E \\
& =A^{\prime} F^{2} e^{-\left(B^{\prime} \phi^{\frac{3}{2}} / F\right)}
\end{aligned}
$$

where $A^{\prime}$ and $B^{\prime}$ are known constants. ${ }^{21}$ This result, known as the Fowler-Nordheim (F-N) equation, predicts a plot of $\ln \left(\mathrm{J}(\mathrm{F}) / \mathrm{F}^{2}\right)$ vs. $1 / F$ will give a straight line with a slope related to the work function $\phi$ of the emitting surface. Use of this equation has provided much of the systematic information now available on the work function of the various faces of pure metals.

These basic ideas of field emission can be used to learn more about the shape and electronic structure of a supported cluster. Information about the shape of the supported cluster comes through a geometrical factor that relates the applied voltage to the electric field $F$ that appears in the equations discussed above. Since the electron emission current depends exponentially on the field strength, and since the field strength is determined by the shape (i.e. radius of curvature) of the supported cluster, small changes in shape produce large changes in the tunneling current. Information about the electronic structure of a supported cluster is contained in $N(\vec{k})$. When considering electron emission from a cluster, the free-electron density $N(\vec{k})$ in Eq. 1 must be replaced with the quantized density of states for the cluster.

\section{Cluster synthesis and sample preparation}

A description of the design and operation of the multiple expansion cluster source (MECS) used in this study can be found in the literature. ${ }^{6,22,4}$ The clusters are grown as aerosol particles in a room temperature inert gas and introduced into a vacuum chamber $\left(10^{-6}\right.$ torr $)$ to form a cluster beam. In each of the experiments described below, cluster samples captured on thin amorphous carbon substrates were analyzed by TEM to determine their distribution in size. Their size distribution studied in this way confirm the ability of the MECS to produce metal clusters having an approximately spherical shape, a controlled mean size, and a narrow size distribution. ${ }^{6,22}$

To perform the field emission studies discussed below, a clean tungsten tip is briefly exposed to the cluster beam until a single cluster is observed by its field emission signature. The tip is then transferred in a vacuum transfer cell at pressures of $\sim 5 \times 10^{-8}$ torr to a UHV field emission apparatus. Experiments are performed in 
the field emission chamber at a pressure of $\sim 2 \times 10^{-10}$ torr.

\section{Size-Dependent Melting of Au Clusters}

In order to investigate the melting transition in nanometer-size clusters, we have developed a technique that uses the field emission current from an individual cluster to measure the melting temperature of that cluster. This approach when coupled with the ability of the MECS to grow clusters of controlled size enables the study of a wide range of cluster sizes ( $\sim 1.0-20 \mathrm{~nm}$ in diameter). Moreover, the sensitivity of the technique appears to be unaffected by cluster size. The details of these measurements have been reported previously ${ }^{8.9}$ and we will not repeat them here. Rather, we will discuss the experimental results for Au clusters within the context of various theoretical treatments of size-dependent melting.

The classical thermodynamic treatment of melting holds that the melting transition occurs when the chemical potentials of the liquid and solid phase are equal. ${ }^{23,24}$ In this context, the melting depression for a small cluster results because the chemical potential for a cluster is modified (compared to the bulk value) by a differential pressure drop across the surface. For a spherical cluster (solid or liquid) of radius $r$, and having a surface tension $\gamma$ the pressure difference is given by ${ }^{25}$

$$
P-P_{\text {ext }}=2 \gamma / r \text {. }
$$

A thermodynamic treatment of the melting temperature of small clusters based on this premise was first proposed by $\mathrm{Pawlow}^{26}$ and has been more recently reevaluated by Hanszen. ${ }^{27}$ This classic analysis of the problem predicts that a reduced plot of $T_{m}(r) / T_{0}$ (where $T_{0}$ is the melting temperature of the bulk solid) vs. the inverse of the cluster radius, $(\mathrm{l} / \mathrm{r})$, will yield a straight line. If the depression in melting temperature is large (as is the case when $r$ is small), then the change in bulk thermodynamic variables with temperature must be taken into account and the straight line predicted by Pawlow becomes slightly curved. ${ }^{28}$ The predictions of this corrected thermodynamic model, are compared to the experimental data for Au clusters in Figure 2. A reasonable fit is obtained provided the cluster radius is greater than $2 \mathrm{~nm} .{ }^{9}$ In fitting the model to the experimental results, $\gamma_{s}$ is the only free parameter that is varied. The two curves in Figure 2 demonstrate the sensitivity of the cluster melting temperature on this parameter.

Other thermodynamic theories for cluster melting exist. For example, Couchman and Jesser ${ }^{29}$ have proposed a dynamic model that treats surface atoms differently than interior atoms. They assume cluster melting occurs via transformation of a surface layer of atoms from solid to liquid (i.e. surface melting) followed by spontaneous growth of this liquid region to consume the entire cluster. The Couchman and Jesser model predicts the same qualitative behavior as the Pawlow model, a monotonic decrease in melting temperature with decreasing cluster size. 
The experimental data obtained by Buffat and Borel, ${ }^{28}$ who studied cluster melting by observing the disappearance of electron diffraction rings from ensembles of $\mathrm{Au}$ clusters (grown by depositing Au atoms on carbon substrates,), are plotted as the shaded area in Figure 2. Although these data are of questionable accuracy as the cluster radius becomes smaller than $\sim 2.5 \mathrm{~nm}$, they seem to indicate a leveling of $T_{m}(r)$ as $r$ becomes small, similar to that measured by field emission. The interesting problem is to theoretically predict this leveling of $T_{m}(r)$.

Several authors have studied cluster melting by means of molecular dynamics (MD) simulation. Most of these studies have been for atoms that interact via a Lennard-Jones 12-6 potential. Using best fit Lennard-Jones parameters for $\mathrm{Au}$, the absolute predictions of these calculations for $T_{m}(r)$ are poor. However, if the $T_{m}(r)$ obtained in the MD simulation is normalized by a $T_{0}$ also obtained by means of MD simulation, the comparison between theory and experiment for $\mathrm{r} \leq 1 \mathrm{~nm}$ is surprisingly good. An example of this agreement is the theoretical calculations of Honeycutt and Anderson ${ }^{30}$ which are plotted on Figure 2.

MD calculations by Ercolessi et al. ${ }^{31}$ and by Jellinek and Garzón ${ }^{32}$ employ empirical potential functions designed to better model the bulk properties of gold. The predictions of these two studies are also plotted on Figure 2. Unfortunately, the calculations of Ercolessi et al. do not extend to clusters with a radius smaller than $1 \mathrm{~nm}$, while the calculations of Garzón and Jellinek were performed for 12-14 atom clusters only. Furthermore, it is not clear that the empirical potentials used by these authors have validity outside of the respective ranges of their calculations.

The experimental data presented in Figure 2 represent a severe test for any empirical potential function of $\mathrm{Au}$. One caution with regard to direct comparison between these experimental data and MD calculations must be noted. The Au clusters studied by field emission were supported on a $\mathrm{W}$ substrate while the MD calculations are typically for free clusters. As cluster size decrease, the effects of cluster-substrate interaction on the melting transition can be significant. Garzón et al. $^{33}$ have simulated the melting of argon clusters on fixed solid lattices and find that $T_{m}$ depends on the strength of the cluster-substrate interaction. Further experiments (with different metals and with a variety of substrate materials) and theoretical calculations, (in the size range below $\mathrm{r} \simeq 2 \mathrm{~nm}$ and for supported as well as free clusters) are clearly needed.

\section{Field Emission Energy Distributions from Au Clusters}

It is useful to consider simple models for the quantized electronic states of a cluster to learn more about the feasibility of detecting these states using field emission. Since, the electronic states of free space (i.e., unsupported) clusters can be described using simple models, systematic case studies illustrating important trends can be made. In what follows, we calculate the expected energy distribution emitted from a cluster 


\section{Reduction in Melting Temperature vs. Cluster Size (Summary of results for Au)}

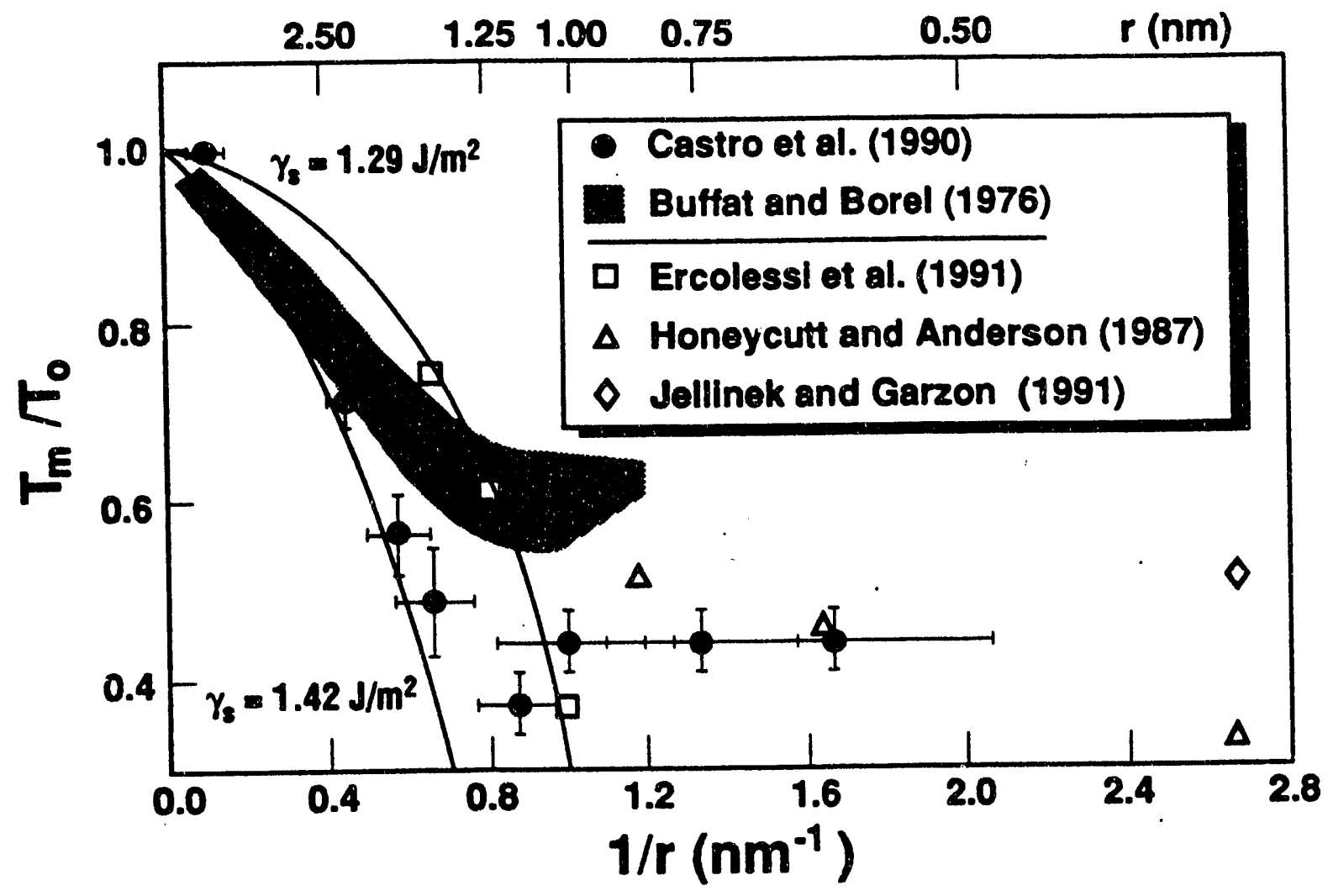

Figure 2: A compilation of the size-dependent melting temperature of supported Au metal clusters. The cluster melting temperature $\left(T_{m}\right)$ is normalized to the bulk melting temperature $\left(T_{0}\right)$ and plotted as a function of $1 / r$ where $r$ is the cluster radius in $\mathrm{nm}$. The solid dots are data obtained from the field emission technique developed by Castro et al. (Ref. 7). The data from Buffet and Borel (Ref. 26) encompass the shaded region. The solid lines are calculated from the modified thermodynamic model for two different values of $\gamma_{s}$ (ref. 7). The results of molecular dynamics calculations by Ercolessi et al.(Ref. 29). Honeycutt and Andreson (Ref. 28), and Jellnick and Garzón (Ref. 30) are also shown. 
on the basis of these ideaiized models and compare this theoretical prediction to a measured energy distribution from a $1 \mathrm{~nm}$ diameter Au cluster. The calculations serve as a useful guide for determining the size of clusters which might show structure in field emission experiments due to their discrete energy states, and the comparison between theory and experiment serves as an indication of how field emission from a single supported cluster can yield valuable information about a cluster's quantized electronic states.

The electronic structure reflected in the mass spectra of alkali clusters ${ }^{34,35}$ indicate the suitability of a shell model for describing cluster states. It is customary to discuss this shell structure using either the infinite square well potential or the infinite harmonic potential well model. In the infinite square well model, the electrons are confined by a potential $\mathrm{V}(\mathrm{r})$ given by

$$
V(r)= \begin{cases}0 & \text { if } r<a \\ \infty & \text { if } r>a\end{cases}
$$

where $\mathbf{a}$ is the radius of the cluster. The radial Schrödinger equation for $r<a$ gives the well known solution

$$
R_{l}(r)=A_{l} j_{l}\left(\frac{\sqrt{2 m E}}{\hbar} r\right)
$$

where $j_{l}$ is the spherical Bessel function. Since this is an infinite well, the eigenfunctions must vanish for $r>a$. Thus, Eq. $\delta$ must equal zero at $r=a$ giving the energies of the n'th shell

$$
E_{n l}=\frac{\hbar^{2} Z_{n l}^{2}}{2 m a^{2}}
$$

where $Z_{n l}$ are the zeros of the spherical Bessel function.

In the infinite harmonic well model, the electrons are confined by a potential $V(r)$ specified by

$$
V(r)=\frac{1}{2} m \omega^{2} r^{2}
$$

where $m$ is the mass of an electron. The parameter $\omega$ is determined for a given cluster by setting the width of the potential well at the vacuum level equal to the diameter of the cluster. A solution of Schrödinger's equation for spherical harmonic oscillator produces complicated eigenfunctions but the eigenvalues are well-known and given by

$$
E_{n l}=\left(2 n+l+\frac{3}{2}\right) \hbar \omega,
$$

where $\mathrm{n}, 1=0,1,2, \cdots$. In general, if $N=2 n+l$, then for $N$ even, there are $\frac{1}{2} N+1$ degenerate solutions while, if $N$ is odd, $\frac{1}{2}(N+1)$ degeneracies occur. 
A realistic potential well for a cluster most likely is an interpolation between the previous examples ${ }^{36}$ and the actual energy levels should lie somewhere between the corresponding levels derived from these simple models. Further elaboration can remove the degeneracies inherent in Eq. 11. For instance, if we introduce a spinorbit coupling term $\alpha \hat{l}$.s. where $\alpha$ is the spin-orbit splitting parameter, we can remove degeneracies. At the present, little is known about realistic values of $\alpha$ to warrant further calculations. Yet another modification is the deviation of the cluster from spherical symmetry. An ellipsoidal shell model was developed for nuclei by Nilsson. ${ }^{37}$ A similar idea can be applied to metal clusters. However, these elaborations will not be applied here.

Based on the electron energy distribution predicted using these two models (Eqs. 9 and 11), we can ascertain the extent that observable structure in the field emission energy distributions can be measured. We find that resolvable splitîngs should be observed for $\mathrm{Au}$ clusters smaller than $\sim 3 \mathrm{am}$. Clusters with diameters smaller than this value should exhibit energy distributions with experimentally resolvable splittings and these distributions will have a shape that deviates significantly from the standard results of field emission theory (see Eq. 3).

An example is shown in Figure 3 where the electron energy distributions predicted for a $1 \mathrm{~nm} \mathrm{Au}$ cluster are compared to experiment. In performing these calculations, the transmission probability through the interface potential barrier was calculated using the Lambin-Vigneron algorithm ${ }^{38,39}$ and combined with the discrete density of states predicted by the models discussed above to yield the field emission energy distributions. These results are compared to the field emission energy distribution obtained from a nominal $1 \mathrm{~nm}$ diameter Au cluster. ${ }^{12}$ The calculated distributions are all convoluted with a gaussian function with a full-width at half-maxirnum of $70 \mathrm{meV}$ to take into account the finite energy resolution of our electron energy analyzer. ${ }^{40}$

These calculations show that for clusters this small, there is a significant deviation from bulk behavior. The calculations based on the two separate shell models for the clusters roughly predict the same structure since the calculated spectrum is strongly weighted by the highest filled states. For clusters in this size range, measurements of the electron energy distribution extending to lower energies are required to distinguish between the two models. At the present time, this structure is not accessible experimentally.

The curve showing results obtained from experiment reveals a number of important differences from the model calculations. First, the entire distribution is shifted below the Fermi energy of the substrate, indicating that the electronic levels of the cluster need not be pinned to the Fermi energy of the substrate. Secund, the significant structure in the energy distribution is observed to shift to lower energies with applied electric field, indicating that a more complete theory involving field penetration into the cluster is required. These observations require modifications to the simple models discussed above which are discussed elsewhere. ${ }^{12,41}$

The most important result indicated by Figure 3 is that the electronic structure 


\section{Energy Distributions for $1 \mathrm{~nm}$ Au Cluster}

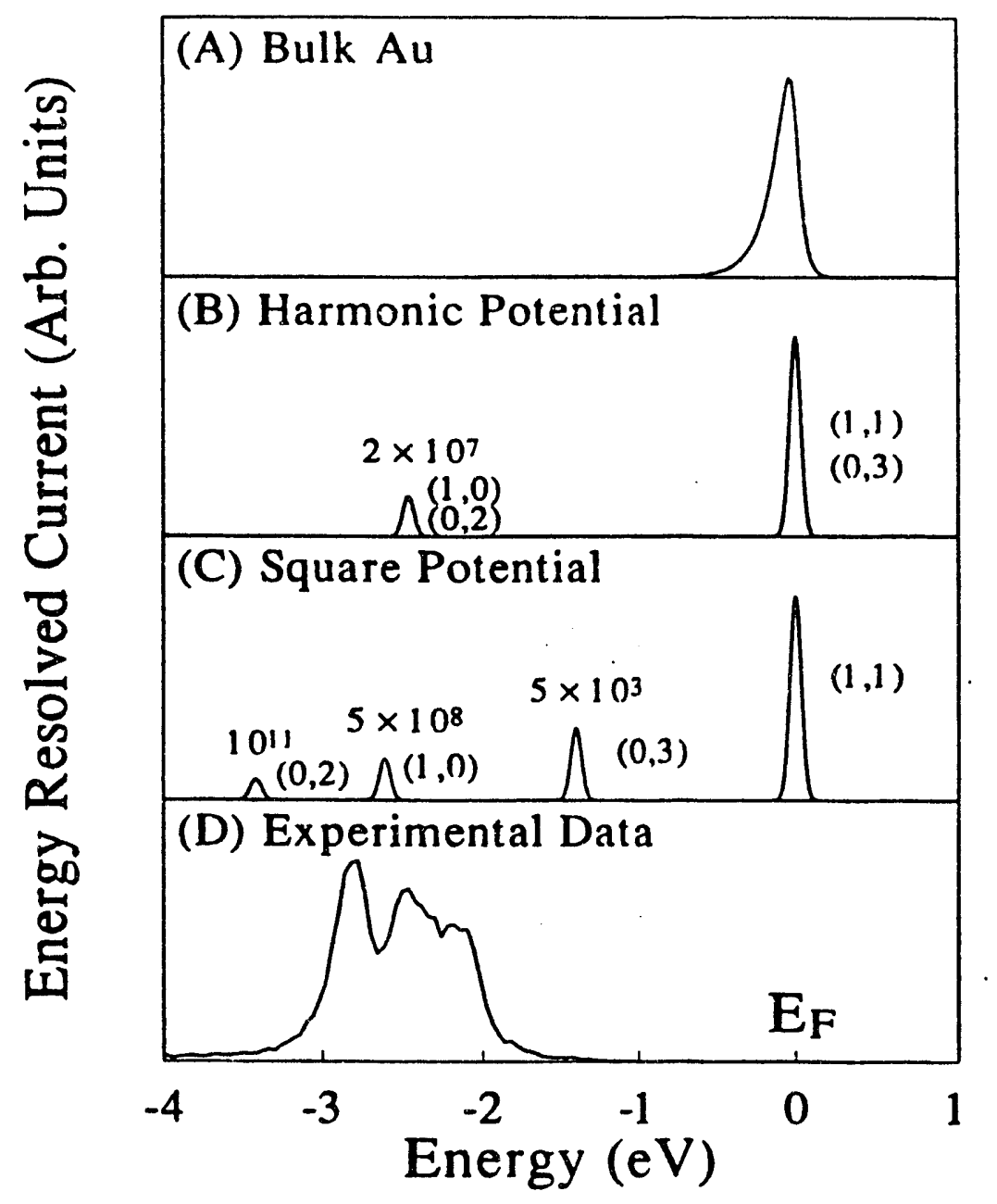

Figure 3: The energy distributions for a $1 \mathrm{~nm}$ diameter Au cluster containing $\sim 40$ atoms. The three calculated curves represent the predictions based on treating the cluster atoms as (a) bulk part of a metal (Eq. 3) with work function $\phi=5.4 \mathrm{eV}$ and an applied electric field of $3 \times 10^{9} \mathrm{~V} / \mathrm{m},(\mathrm{b})$ a separate species described by the infinite harmonic model, and (c) a separate species described by the infinite square potential discussed above. The three model calculations are all plotted with their highest filled states set equal to $E_{F}$. Before plotting, the features at low energy were magnified by the multiplicative factors shown. The $(n, l)$ values for each feature are also indicated. In (d), an experimental data curve obtained from a nominal $1 \mathrm{~nm} \mathrm{Au}$ cluster supported on a W substrate is also shown. The zero of energy for this curve is the Fermi energy of the substrate. 
of a supported nanometer-size cluster can now be probed. Studies performed as a function of cluster size can be undertaken on a wide variety of different substrates and for different regimes of coupling between the substrate and cluster. We believe that a systematic exploitation of this technique will provide an important set of data regarding the factors that influence the quantized electronic structure of small supported clusters.

\section{Summary}

We have reviewed some of the results that have been recently obtained on individual metal clusters of controlled size that were supported on a substrate. The experiments summarized above employed a multiple expansion cluster source in conjunction with field ernission microscopy techniques. The results of this work indicate that detailed information about the size-dependent melting and electronic structure of nanometersize supported clusters can be obtained.

The utility of this approach was illustrated by two examples. The size-dependent melting temperature of Au clusters has been measured and was discussed within the context of existing models for the melting transition. The energy distribution emitted from a $1 \mathrm{~nm} \mathrm{Au}$ cluster has been measured and was compared to simple model predictions.

In conclusion, based on the results already obtained, it now seems feasible to consider a sequence of experiments in which free-space clusters of known size and composition are systematically prepared and studied on a wide variety of different substrates to learn more about the structural, thermodynamic, and electronic properties of individual, supported clusters.

\section{Acknowledgements}

This work was partially supported by the U.S. Department of Energy under grant DE-FG02-88ER45162. M.E. Lin thanks the David Ross Foundation of Purdue University for financial support during the course of this study. A. Ramachandra thanks the Dow Chemical Corporation and Arco for financial support during the course of this study.

\section{References}

[1] R.P. Andres, R.S. Averback, W.L. Brown, L.E. Brus, W.A. Goddard, A. Kaldor, S.G. Louie, M. Moscovits, P.S. Peercy, S.J. Riley, R.W. Siegel, F. Spaepen, and Y. Wang, J. Mater. Res. 4, 704 (1989).

[2] R.W. Siegel, MRS Bulletin XV; No. 10, 60 (1990). 
[3] R.S. Averback, J. Bernholc, and D.L. Nelson, editors, Clusters and ClusterAssembled Materials, Materials Research Society; Vol. 206, Pittsburgh, PA, 1991.

[4] T. Castro, Y.Z. Li, R. Reifenberger, E. Choi, S.B. Park, and R.P. Andres, J. Vac. Sci. Technol. A7, 2845 (1989).

[5] T. Castro, E. Choi, Y.Z. Li, R.P. Andres, and R. Reifenberger, Proc. Mat. Res. Soc. 206, 159 (1991).

[6] S.B. Park, PhD thesis, 1988, Purdue University ( «npublished).

[7] T. Castro, PhD thesis, 1989, Purdue University (unpublished).

[8] T. Castro, R. Reifenberger, E. Choi, and R.P. Andres, Surf. Sci. 234, 43 (1990).

[9] T. Castro, R. Reifenberger, E. Choi, and R.P. Andres, Phys. Rev. B 42, 8548 (1990).

[10] Y.Z. Li, PhD thesis, 1989, Purdue University (unpublished).

[11] Y.Z. Li, R. Reifenberger, E. Choi, and R.P. Andres, Surf. Sci. 250, 1 (1991).

[12] M.E. Lin, R.P. Andres, and R. Reifenberger, Phys. Rev. Lett. 67, 477 (1991).

[13] J. Hölz] and F.K. Schulte, Work function of metals, in Springer Tracts in Modern Physics, Vol 85, edited by G. Hohler, Springer Verlag, 1979.

[14] R. Difoggio and R. Gomer, Phys. Rev. B 25, 3490 (19ع2).

[15] C. Dharmadhikari and R. Gomer, Surf. Sci. 143, 223 (1984).

[16] J.W. Gadzuk and E.W. Plummer, Rev. Mod. Phys. 43, 487 (1973).

[17] D. Venus and M.J.G. Lee, Surf. Sci. 125, 452 (1983).

[18] Y. Gao and R. Reifenberger, Phys. Rev. B35, 8301 (1987).

[19] A... Melmed, J. Vac. Sci. Technol. B9, 601 (1991).

[20] A. Modinos, Field, Thermionic and Secondary Electron Emission Spectroscopy, Plenum Press, New York, 1984.

[21] R.H. Fowler and L.W. Nordheim, Proc. Roy. Soc. Lond. A119, 173 (1928).

[22] E. Choi and R.P Andres, in Physics and Chemistry of Small Clusters, edited by P. Jena, B.K. Rao, and S.N. Khanna, page 61, Plenum Press, New York, 1987.

[23] Charles Kittel and Herbert Kroemer, Thermal Physics, W.H. Freeman and Company, 1980.

[24! r. Reif, Fundamentals of Statistical and Thermal Physics, McGraw-Hill, 1965. 
[25] I. Prigogine R. Defay and A. Bellemans, Surface Tension and Adsorption, John Wiley and Sons Inc., 1966.

[26] P. Pawlow, Z. Phys. Chem. 65, 1 (1909).

[27] K. J. Hanszen, Z. Phys. 157, 523 (1960).

[28] Ph Buffat and J.P. Borel, Phys. Rev. A13, 2287 (1976).

[29] P.R. Couchman and W.A. Jesser, Nature 269, 481 (1977).

[30] J.D. Honeycutt and H.C. Anderson, J. Phys. Chem. 91, 4950 (1987).

[31] F. Ercolessi, W. Andreoni, and E. Tosatti, Phys. Rev. Lett. 66, 911 (1991).

[32] J. Jellinek and I.L. Garzón, Z. Phys. D 20, 235 (1991).

[33] I.L Garzon et al., in Physics and Chemistry of Small Clusters, edited by P. Jena, B.K. Rao, and S.N. Khanna, page 193, Plenum Press, New York, 1987.

[34] W.D. Knight, K. Clemenger, W.A. de Heer, W.A: Saunders, M.Y. Chou, and M.L. Cohen, Phys. Rev. Lett. 52, 2141 (1984).

[35] W.D. Knight, W.A. de Heer, K. Clemenger, and W.A. Saunders, Solid State Commun. 53, 445 (1985).

[36] M. G. Mayer and J. H. D. Jensen, Elementary Theory of Nuclear Shell Structure, John Wiley and Sons Inc., 1955.

[37] S.F. Nilsson, Mat.-Fys. Medd. Dan. Vidensk. Selsk. 29, 1 (1955).

[38] Ph Lambin and J P Vigneron, J. Phys. A: Math. Gen. 14, 1815 (1981).

[39] J P Vigneron and Ph Lambin, J. Phys. A: Math. Gen. 13, 1135 (1980).

[40] Y. Gao, R. Reifenberger, and R.M. Kramer, J. Phys. E: Sci. Instrum. 18, 381 (1985).

[41] M.E. Lin, R.P. Andres, and R. Reifenberger, (to be published) (1991). 

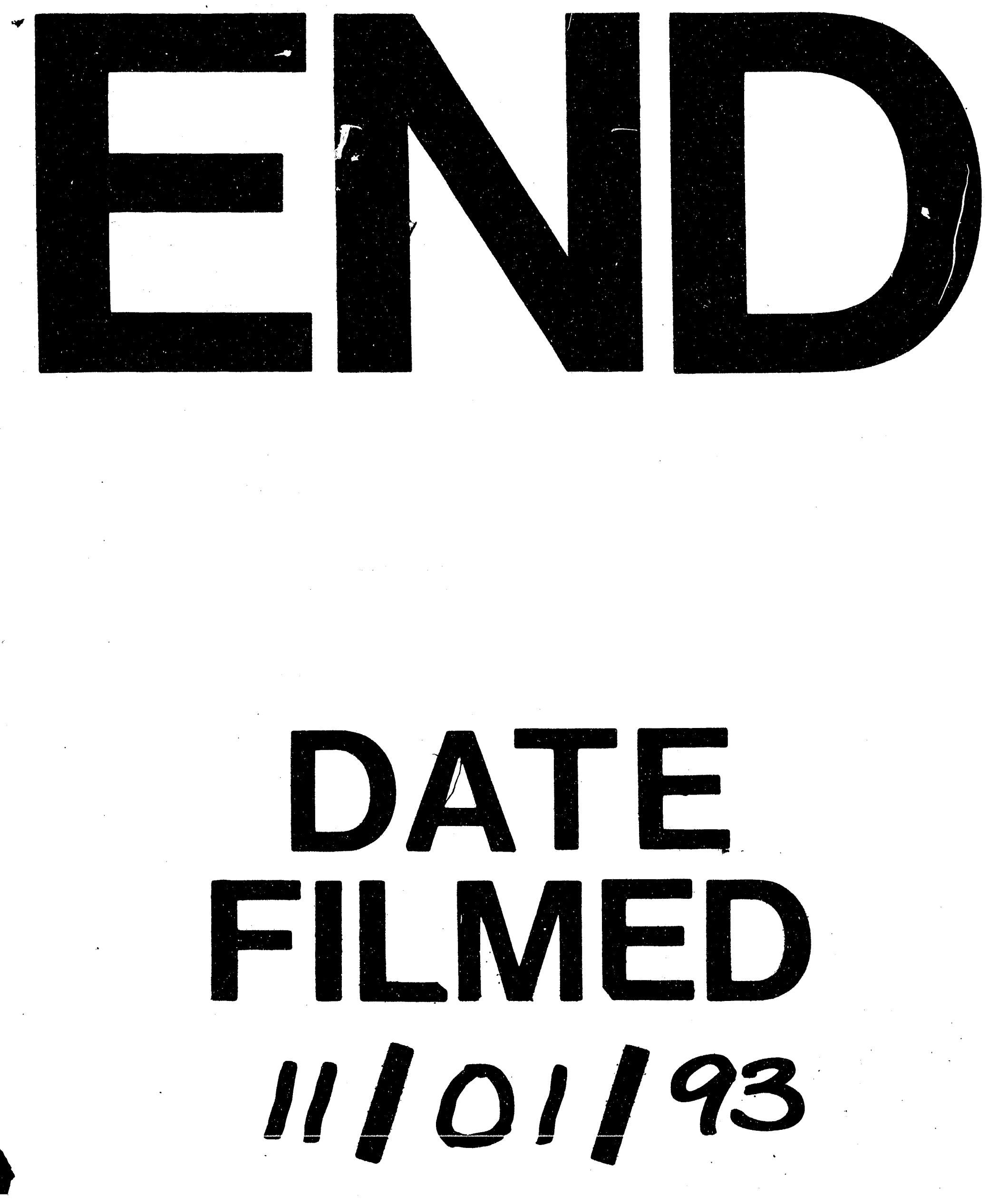
